\title{
Identification of a Novel Variant of ARHGAP29 in a Chinese Family with Nonsyndromic Cleft Lip and Palate
}

\author{
Jian-Xia Tang, ${ }^{1}$ Xiang-Shui Xiao, ${ }^{1}$ Kai Wang, ${ }^{2}$ Jie-Yuan Jin, ${ }^{3}$ Liang-Liang Fan $\mathbb{D},{ }^{3,4}$ \\ and Rong Xiang $\mathbb{1}^{3,4}$ \\ ${ }^{1}$ Department of Prosthodontics, Xiangya Stomatological Hospital \& School of Stomatology, Central South University, Changsha, \\ Hunan, China \\ ${ }^{2}$ Department of Oral and Maxillofacial Surgery, The Second Xiangya Hospital, Central South University, Changsha, Hunan, China \\ ${ }^{3}$ School of Life Sciences, Central South University, Changsha, China \\ ${ }^{4}$ Human Key Laboratory of Animal Models for Human Diseases, School of Life Sciences, Central South University, Changsha, China
}

Correspondence should be addressed to Liang-Liang Fan; swfanliangliang@csu.edu.cn and Rong Xiang; shirlesmile@csu.edu.cn

Received 4 May 2020; Revised 27 September 2020; Accepted 30 September 2020; Published 23 October 2020

Academic Editor: Marco Fichera

Copyright (C) 2020 Jian-Xia Tang et al. This is an open access article distributed under the Creative Commons Attribution License, which permits unrestricted use, distribution, and reproduction in any medium, provided the original work is properly cited.

Background. Cleft lip with or without cleft palate $(\mathrm{CL} / \mathrm{P})$ is the most common facial birth defect, with a worldwide incidence of 1 in 700-1000 live births. CL/P can be divided into syndromic CL/P (SCL/P) and nonsyndromic CL/P (NSCL/P). Genetic factors are an important component to the etiology of NSCL/P. ARHGAP29, one of the NSCL/P disease-causing genes, mediates the cyclical regulation of small GTP binding proteins such as RhoA and plays an essential role in cellular shape, proliferation, and craniofacial development. Methods. The present study investigated a Chinese family with NSCL/P and explored potential pathogenic variants using whole-exome sequencing (WES). Variants were screened and filtered through bioinformatic analysis and prediction of variant pathogenicity. Cosegregation was subsequently conducted. Results. We identified a novel heterozygous missense variant of ARHGAP29 (c.2615C > T, p.A872V) in a Chinese pedigree with NSCL/P. Conclusion. We detected the disease-causing variant in this NSCL/P family. Our identification expands the genetic spectrum of ARHGAP29 and contributes to novel approaches to the genetic diagnosis and counseling of CL/P families.

\section{Introduction}

Cleft lip with or without cleft palate (CL/P) is one of the most prevalent human birth defects, with a worldwide incidence of 1 in 700-1000 live births [1]. The prevalence varies with ethnicity, sex, and cleft type [2]. According to whether patients have other organ malformations, $\mathrm{CL} / \mathrm{P}$ is divided into syndromic CL/P (SCL/P) and nonsyndromic CL/P (NSCL/P). Although $\mathrm{CL} / \mathrm{P}$ is found in more than 500 syndromes (including van der Woude syndrome 1 [OMIM_119300], ectrodactyly, ectodermal dysplasia, and cleft lip/palate syndrome 3 [OMIM_604292]), NSCLP preponderates in congenital facial cleft cases [3-5].

SCL/P follows Mendelian inheritance with diseasecausing genes including IRF6, TP63, TBX1, and SPECC1L [5-8]. In contrast, NSCL/P is thought to have a complex etiology, with genetic factors acting in concert with environmental effects, which leads to variable phenotypes and incomplete penetrance $[3,9]$. With the increasing availability of genome-wide association studies (GWAS) and wholeexome sequencing (WES), many genes have been identified as NSCL/P causative genes, including CTNND1, PLEKHA5, PLEKHA7, CDH1, and ARHGAP29 [3, 10, 11].

ARHGAP29 is located on 1p22.1 and encodes the Rho GTPase activating protein (GAP) 29. RAP1 is a small GTPase that regulates Rho GTPase signaling. ARHGAP29 and RAP1 effectors (RADIL and RASIP1) translocate to the plasma membrane, where they form a multiprotein complex mediating RAP1-induced inhibition of Rho signaling [12]. As a GTPase activator for the Rho-type GTPases by converting them to an inactive GDP-bound state, ARHGAP29 has a strong activity toward RhoA to suppress RhoA signaling 


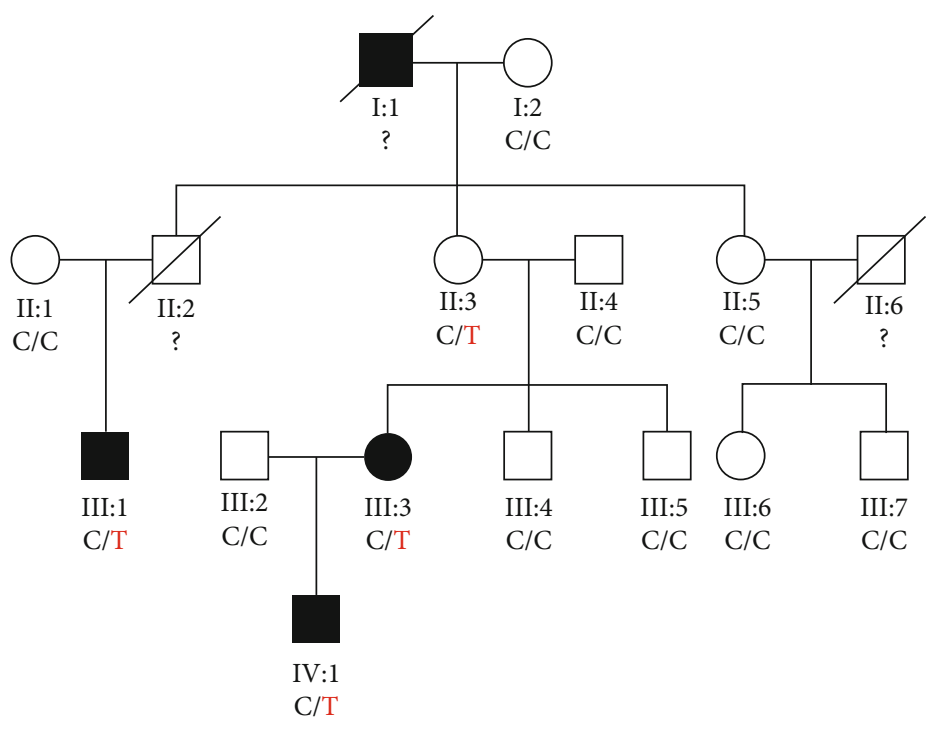

(a)

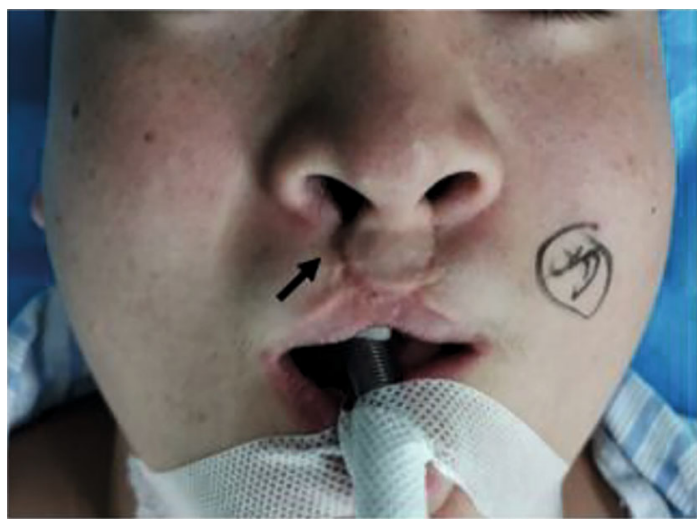

(b)

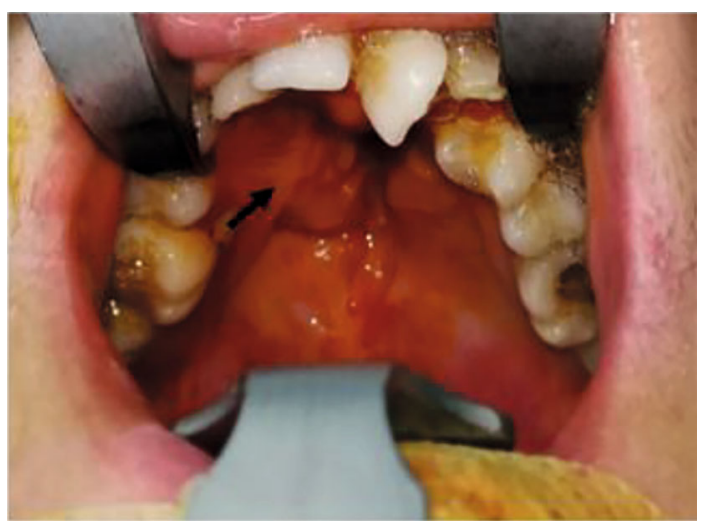

(c)

Figure 1: (a) Pedigree of the CL/P family with segregation analysis. The black symbols represent an affected member, and the arrow indicates the proband. Genotypes are identified by letters and slash, with red representing the variant. (b, c) The orofacial phenotypes of the proband. The proband has the scar due to the cleft lip repair (b), palate fistula, and altered dentition (c).

and dampen ROCK and MYH9 activities in endothelial cells. Rho signaling plays an essential role in cellular shape, movement, cell-cell interactions, proliferation, and craniofacial development [13]. Hence, ARHGAP29 mutations could lead to NSCL/P. In fact, ARHGAP29 mutations have been shown to impede oral adhesions during orofacial development in mice.

In this study, we reported a NSCL/P family from Hunan province, China. We identified a novel missense variant of ARHGAP29 (c.2615C > T, p.A872V) in the proband, which was inherited from his affected mother. To the best of our knowledge, this variant has not been reported in previous studies or presented in various single nucleotide polymorphism (SNP) databases.

\section{Material and Methods}

2.1. Patients and Subjects. The Review Board of Xiangya Stomatological Hospital of the Central South University approved this research (approval number 20190038). 51 $\mathrm{CL} / \mathrm{P}$ patients were recruited, and 10 were selected to undergo WES according to family history and disease severity. Written informed consent was obtained from patients and their guardians, in which all subjects are consented to this study and the publication of the images. Blood was collected from the proband and related family members. Segregation analysis was performed in all family members based on the WES results.

2.2. Whole-Exome Sequencing. Genomic DNA was extracted with the DNeasy Blood and Tissue Kit (Qiagen, Valencia, Calif., USA). The Berry Genomics Co., Ltd. (Chengdu, China) provided the exome capture, high-throughput sequencing, and common variant filtering. The clustering of the indexcoded samples was performed on the cBot Clster Generation System and Hiseq PE Cluster Kit (Illumina) according to the manufacturer's instructions. After cluster generation, the DNA libraries were sequenced on the Illumina Hiseq platform, and $150 \mathrm{bp}$ paired-end reads were generated. After filtering the common variants (frequency $\geq 0.05$ ) using the 1000 Genomes Project database (https://www.genome.gov/ 27528684/1000-genomes-project/), the Chinese Millionome 


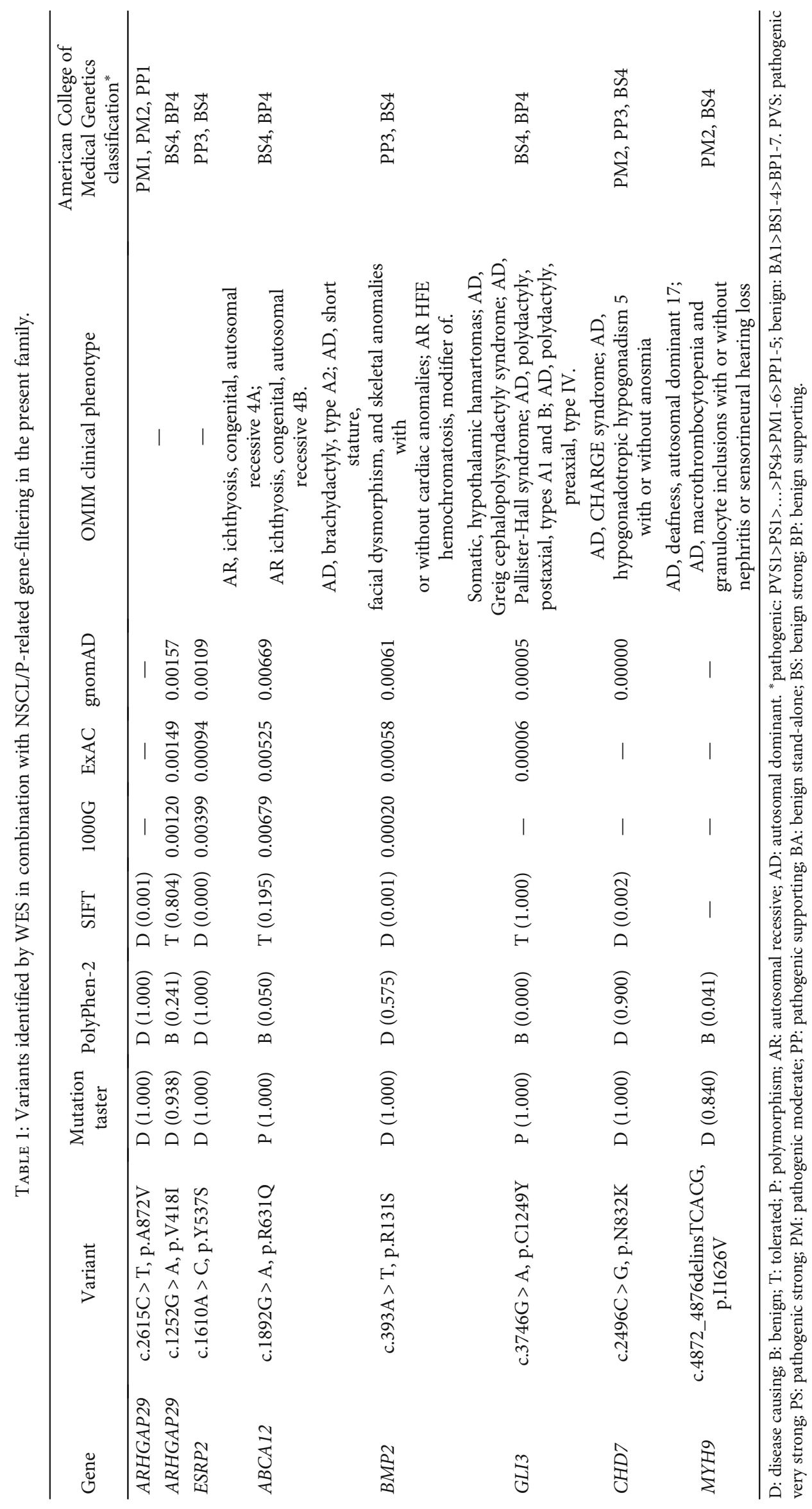




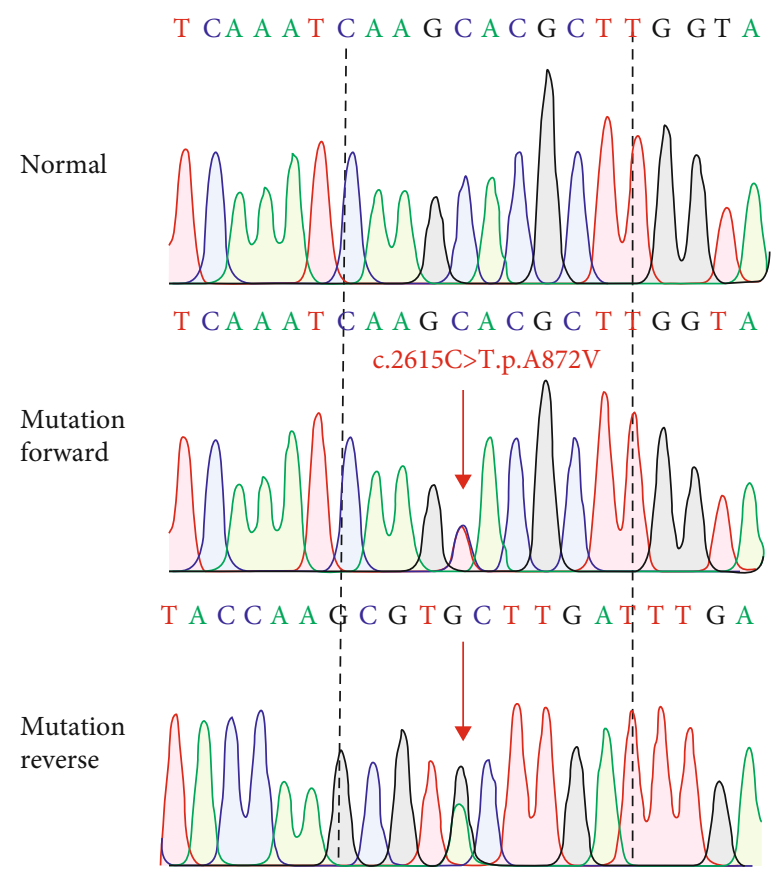

(a)

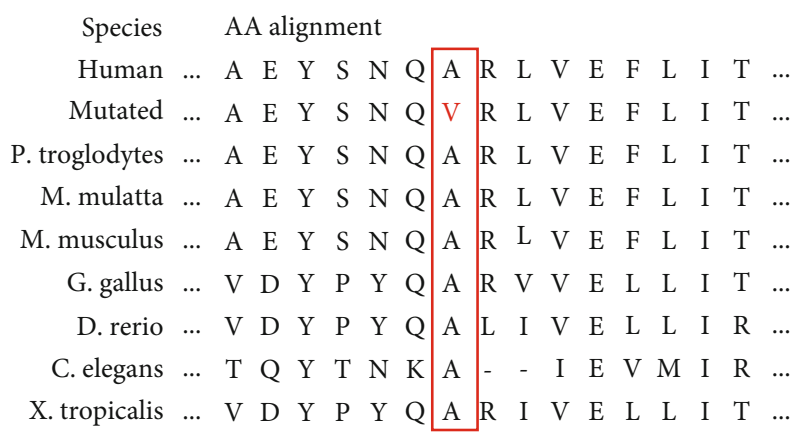

(b)

interaction

\section{Rap2 interaction \\ C1 RhoGA

$$
\text { A872V }
$$

(c)

FIgURE 2: (a) The sequencing results of the ARHGAP29 variant. Sequence chromatograms indicate the heterozygous variant (c.2615C $>$ T, p.A872V) in the CL/P family. (b) The mutated site (A872V) is highly evolutionary conserved cross species. The red graphic represents a mutated amino acid, and the red box emphasizes cross species comparison. (c) A schematic diagram of the ARHGAP29 structure and the mutated AA site (A872V). Gray boxes represent the domains. "Rap2 interaction" indicates a coiled-coil region known to interact with Rap2; "C1" indicates a C1 domain; "RhoGAP" indicates a Rho GTPase domain; "PTPL1 interaction" indicates a small C-terminal region that interacts with PTPL1; and the black arrow represents the mutated AA site.

Database (https://db.cngb.org/cmdb/), the Genome Aggregation database (http://gnomad.broadinstitule.org), and the Exome Aggregation Consortium database (http://exac .broadinstitute.org/), unique single-nucleotide polymorphisms (SNPs) were identified. Potential causative variants were screened by the list of genes related to NSCL/P (Table S1) and then predicted using bioinformatic programs including MutationTaster (http://www.mutationtaster.org/), Polyphen-2 (http://genetics.bwh.harvard.edu/pph2/), and SIFT (http://provean.jcvi.org/index.php). The analyses of gene function, inheritance pattern, and clinical phenotype were conducted using Online Mendelian Inheritance in Man (OMIM) (https://www.omim.org).

2.3. Cosegregation Analysis. Primer pairs were designed via DNASTAR. The primers sequences will be provided upon request (ARHGAP29 c.2615C > T f: CAGGGTAGTAGATC ATGCAGAAG; ARHGAP29 c.2615C > T r: GGTGATAAC AGAGGCTTTGGA). The target fragments were amplified via polymerase chain reaction (PCR) and analyzed using the ABI 3100 Genetic Analyzer (ABI, Foster City, CA).

\section{Results}

3.1. Clinical Features. We collected $10 \mathrm{CL} / \mathrm{P}$ families to screen for mutations by WES and identified the genetic lesion for one family. This family was from the Hunan Province, China (Figure 1(a)). The proband (II:1), a 16-year-old boy, was admitted to our hospital for a second palate fistula operation. The proband was diagnosed with cleft lip and palate at birth and had palate fistula and altered dentition without other organic abnormities (Figures $1(\mathrm{~b})$ and $1(\mathrm{c})$ ). The proband underwent lip repair surgery in the local hospital when he was 3 years old. His mother (III:3), uncle (III: 1), and grandfather $(\mathrm{I}: 1)$ had CL/P. Other family members were unaffected.

3.2. Genetic Analysis. WES yielded $12.1 \mathrm{~Gb}$ of data with $98.22 \%$ coverage of the target region and $97.54 \%$ of the target covered over $10 \times$. After a series of database analyses and filtering, 892 unique SNPs were detected in the proband. The variants were filtered by NSCL/P genes (Table S1), and a set of eight heterozygous variants in seven genes in the proband was identified (Table 1). By analyzing the bioinformatic prediction, inheritance pattern, OMIM clinical phenotypes, 
and American College of Medical Genetics classification [14] of these eight genes, we suspected one of the ARGHAP29 variants (c.2615C $>\mathrm{T}, \mathrm{p} . \mathrm{A} 872 \mathrm{~V})$ to be the causative variant in the family.

Sanger sequencing results indicated that the ARHGAP29 variant (c.2615C > T, p.A872V) (Figure 2(a)) in the proband was inherited from his mother. Further sequencing in all subjects showed that this variant only existed in all affected subjects (III:1, III:3, and IV:1) and II:3. Additionally, the amino acid sequence alignment analysis suggested that the altered site was located in a highly evolutionarily conserved site (Figure 2(b)). Therefore, we considered the ARHGAP29 variant (c.2615C $>\mathrm{T}, \mathrm{p} . \mathrm{A} 872 \mathrm{~V}$ ) to be the main pathogenic factor in this family.

\section{Discussion}

$\mathrm{CL} / \mathrm{P}$ is one of the most common developmental deformities with an incidence rate of $1.67 \%$ in China, of which approximately $70 \%$ cases are NSCL/P [15]. In NSCL/P cases, $80 \%$ are sporadic and $20 \%$ are familial cases [16]. Although $\mathrm{NSCL} / \mathrm{P}$ is associated with various factors and not attributed to single etiologic mechanism, mutation screening in $\mathrm{CL} / \mathrm{P}$ families could contribute to our understanding of genetic factor influence [17]. Pathogenic or likely pathogenic variants are identified in approximately $14 \%$ of multigenerational families with moderate to high penetrance [3]. In the present study, we reported a NSCL/P family across four generations with moderate penetrance. We confirmed presence of a heterozygous ARHGAP29 variant (c.2615C > T, p.A872V) in all patients. Notably, II:3 also harbored this variant, but was unaffected; however, incomplete penetrance is common in NSCL/P families. Regrettably, we did not test the variant of I: 1 and II:2, who were considered to carry this base alteration, to further confirm the genotype-phenotype correlation.

$\mathrm{CL} / \mathrm{P}$ results from facial morphogenesis and tissue fusion anomalies during embryonic development [13]. ARHGAP29 is a mediator of RhoA signaling that is related to cellular movement and proliferation in craniofacial development. Hence, ARHGAP29 defects are associated with CL/P. The structure of ARHGAP29 includes four domains: a coiledcoil region known to interact with Rap2, a C1 domain, the Rho GTPase domain, and a small C-terminal region that interacts with PTPL1 (Figure 2(c)) [18]. The alignment of the Rho GTPase domain covering $669^{\text {th }}-881^{\text {st }}$ amino acids (AAs) in ARHGAP29 is a highly conserved region that contains a catalytic residue and seven residues that compose the putative GTPase interaction site [9]. Ala at position 872 is one of putative GTPase interaction sites, and Val substitution at this site may thus affect the structure and function [9].

We summarized all known ARHGAP29 mutations [9, 10, 18-21]. 19 ARHGAP29 mutations have been reported in previous studies (Table 2). 18 mutations were identified in CL/P patients, and only one was detected in cleft palate $(\mathrm{CP})$, suggesting ARHGAP29 is highly associated with $\mathrm{CL} / \mathrm{P}$. In addition, incomplete penetrance of $\mathrm{CL} / \mathrm{P}$ families with ARHGAP29 mutations has been previously reported and confirmed in the present study. The moderate penetrance suggests that other factors play a role in $\mathrm{CL} / \mathrm{P}$ occurrence,
TABLE 2: Point mutations of ARHGAP29 causing cleft in patients.

\begin{tabular}{|c|c|c|c|}
\hline Mutation & Inheritance & Phenotypes & PMID \\
\hline c.62_63delCT, p.S21YfsX20 & $\mathrm{AD}$ & NSCLP & 23008150 \\
\hline c.76A > G, p.T26A & $\mathrm{AD}$ & NSCL & 23008150 \\
\hline c. $94 \mathrm{~A}>\mathrm{T}, \mathrm{p} . \mathrm{K} 32 \mathrm{X}$ & $\mathrm{AD}$ & NSCLP & 25512736 \\
\hline c. $137 \mathrm{~A}>\mathrm{G}, \mathrm{p} . \mathrm{K} 46 \mathrm{R}$ & $\mathrm{AD}$ & NSCLP & 23008150 \\
\hline c. $698-1 G>C$ & IP & NSCL/P & 27350171 \\
\hline c.976A > T, p.K326X & $\mathrm{AD}$ & NSCLP & 23008150 \\
\hline c. $1475 \mathrm{C}>\mathrm{A}, \mathrm{p} . \mathrm{S} 492 \mathrm{X}$ & IP & NSCL/P & 27350171 \\
\hline c. $1576+1 \mathrm{G}>\mathrm{A}$ & IP & NSCL/P & 27350171 \\
\hline c. $1654 \mathrm{~T}>\mathrm{C}, \mathrm{p} . \mathrm{S} 552 \mathrm{P}$ & $\mathrm{AD}$ & $\mathrm{CP}$ & 28029220 \\
\hline c. $1865 \mathrm{C}>\mathrm{T}, \mathrm{p} . \mathrm{T} 622 \mathrm{M}$ & $\mathrm{AD}$ & NSCLP & 23008150 \\
\hline c.1939C > T, p.R647X & $\mathrm{AD}$ & $\mathrm{CL} / \mathrm{P}$ & 25704602 \\
\hline c. $2109+1 \mathrm{G}>\mathrm{A}$ & $\mathrm{AD}$ & NSCL/P & 27350171 \\
\hline c. $2367 \mathrm{G}>\mathrm{A}, \mathrm{p} . \mathrm{W} 789 \mathrm{X}$ & $\mathrm{AD}$ & $\mathrm{CL} / \mathrm{P}$ & 25704602 \\
\hline c. $2494 \mathrm{G}>$ A, p.A832T & $\mathrm{AD}$ & NSCLP & 23008150 \\
\hline c. $2533 \mathrm{~A}>$ G, p.I845V & $\mathrm{AD}$ & NSCLP & 23008150 \\
\hline c. $2615 \mathrm{C}>\mathrm{T}, \mathrm{p} . \mathrm{A} 872 \mathrm{~V}$ & IP & NSCLP & \\
\hline c. $2738 \mathrm{C}>\mathrm{A}, \mathrm{p} . \mathrm{S} 913 \mathrm{~L}$ & $\mathrm{AD}$ & CL & 25081408 \\
\hline c.2864G > A, p.R955H & $\mathrm{AD}$ & NSCLP & 23008150 \\
\hline c.3118G > T, p.G1040X & $\mathrm{AD}$ & $\mathrm{CL} / \mathrm{P}$ & 25704602 \\
\hline c. $3425 \mathrm{G}>\mathrm{A}, \mathrm{p} . \mathrm{R} 1142 \mathrm{Q}$ & $\mathrm{AD}$ & NSCLP & 23008150 \\
\hline
\end{tabular}

Red word indicates the case in the present study. AD: autosomal dominant; IP: confirmed incomplete penetrance; NSCLP: nonsyndromic cleft lip and palate; NSCL: nonsyndromic cleft lip; NSCL/P: nonsyndromic cleft lip with or without cleft palate; $\mathrm{CP}$ : cleft palate; CL/P: cleft lip with or without cleft palate; CL: cleft lip.

for example, environmental factors. Similarly, p.A832T and p.I845V in the Rho GTPase domain demonstrate the significant impact of this domain alteration for orofacial development [9].

\section{Conclusions}

In summary, the present study identified a novel heterozygous missense variant (c.2615C $>\mathrm{T}, \mathrm{p} . \mathrm{A} 872 \mathrm{~V})$ of $A R H-$ GAP29 in a Chinese family with CL/P. The identification expands the spectrum of known ARHGAP29 mutations, further demonstrates the association of ARHGAP29 and CL/P, and may contribute to novel approaches to the genetic diagnosis and counseling of CL/P families.

\section{Data Availability}

The datasets used and/or analyzed during the current study are available from the corresponding author upon reasonable request.

\section{Conflicts of Interest}

The authors declare that they have no conflicts of interest. 


\section{Acknowledgments}

We thank the proband and his family members for their participation in this study and all patient advisers for their assistance in clinical examination and blood specimen collection. This work was supported by the National Science and Technology Major Project of the Ministry of Science and Technology of China (2017ZX10103005-006), the National Natural Science Foundation of China (81970403 and 82000427), National Natural Science Foundation of Hunan province (2020JJ5785), and the Emergency Project of Prevention and Control for COVID-19 of Central South University (160260003).

\section{Supplementary Materials}

Supplementary Table S1. Causative/potential causative genes related to NSCL/P. (Supplementary Materials)

\section{References}

[1] M. J. Dixon, M. L. Marazita, T. H. Beaty, and J. C. Murray, "Cleft lip and palate: understanding genetic and environmental influences," Nature Reviews. Genetics, vol. 12, no. 3, pp. 167-178, 2011.

[2] P. M. Good, J. B. Mulliken, and B. L. Padwa, "Frequency of Le fort I osteotomy after repaired cleft lip and palate or cleft palate," The Cleft Palate-Craniofacial Journal, vol. 44, no. 4, pp. 396-401, 2017.

[3] L. L. Cox, T. C. Cox, L. M. Moreno Uribe et al., "Mutations in the epithelial cadherin-p120-catenin complex cause Mendelian non-syndromic cleft lip with or without cleft palate," American Journal of Human Genetics, vol. 102, no. 6, pp. 1143-1157, 2018.

[4] T. J. Wang, K. S. Hsieh, J. P. Lai, M. H. Tsai, Y. C. Liang, and Y. H. Chang, "Novel mutations of IRF6 gene in Taiwanese Van der Woude syndrome patients," Pediatrics and Neonatology, vol. 60, no. 2, pp. 218-220, 2019.

[5] E. Soares, Q. Xu, Q. Li et al., "Single-cell RNA-seq identifies a reversible mesodermal activation in abnormally specified epithelia of p63 EEC syndrome," Proceedings of the National Academy of Sciences of the United States of America, vol. 116, no. 35, pp. 17361-17370, 2019.

[6] L. Maili, A. Letra, R. Silva et al., "PBX-WNT-P63-IRF6 pathway in nonsyndromic cleft lip and palate," Birth Defects Research, vol. 112, no. 3, pp. 234-244, 2019.

[7] N. Funato and H. Yanagisawa, "Deletion of the T-box transcription factor gene, Tbx1, in mice induces differential expression of genes associated with cleft palate in humans," Archives of Oral Biology, vol. 95, pp. 149-155, 2018.

[8] E. J. Bhoj, D. Haye, A. Toutain et al., "Phenotypic spectrum associated with SPECC1L pathogenic variants: new families and critical review of the nosology of Teebi, Opitz GBBB, and Baraitser-Winter syndromes," European Journal of Medical Genetics, vol. 62, no. 12, p. 103588, 2019.

[9] E. J. Leslie, M. A. Mansilla, L. C. Biggs et al., "Expression and mutation analyses implicate ARHGAP29 as the etiologic gene for the cleft lip with or without cleft palate locus identified by genome-wide association on chromosome 1p22," Birth Defects Research. Part A, Clinical and Molecular Teratology, vol. 94, no. 11, pp. 934-942, 2012.
[10] H. Liu, T. Busch, S. Eliason et al., "Exome sequencing provides additional evidence for the involvement of ARHGAP29 in Mendelian orofacial clefting and extends the phenotypic spectrum to isolated cleft palate," Birth Defects Research, vol. 109, no. 1, pp. 27-37, 2017.

[11] R. Ittiwut, C. Ittiwut, P. Siriwan, V. Chichareon, K. Suphapeetiporn, and V. Shotelersuk, "Variants of theCDH1(E-Cadherin) gene associated with oral clefts in the Thai population," Genetic Testing and Molecular Biomarkers, vol. 20, no. 7, pp. 406-409, 2016.

[12] A. Post, W. J. Pannekoek, B. Ponsioen, M. J. Vliem, and J. L. Bos, "Rap1 spatially controls ArhGAP29 to inhibit rho signaling during endothelial barrier regulation," Molecular and Cellular Biology, vol. 35, no. 14, pp. 2495-2502, 2015.

[13] P. A. Mossey, J. Little, R. G. Munger, M. J. Dixon, and W. C. Shaw, "Cleft lip and palate," Lancet, vol. 374, no. 9703, pp. 1773-1785, 2009.

[14] S. Richards, N. Aziz, S. Bale et al., "Standards and guidelines for the interpretation of sequence variants: a joint consensus recommendation of the American College of Medical Genetics and Genomics and the Association for Molecular Pathology," Genetics in Medicine, vol. 17, no. 5, pp. 405-423, 2015.

[15] D. Fan, S. Wu, L. Liu et al., "Prevalence of non-syndromic orofacial clefts: based on 15,094,978 Chinese perinatal infants," Oncotarget, vol. 9, no. 17, pp. 13981-13990, 2018.

[16] M. Basha, B. Demeer, N. Revencu et al., "Whole exome sequencing identifies mutations in $10 \%$ of patients with familial non-syndromic cleft lip and/or palate in genes mutated in well-known syndromes," Journal of Medical Genetics, vol. 55, no. 7, pp. 449-458, 2018.

[17] T. H. Beaty, M. L. Marazita, and E. J. Leslie, "Genetic factors influencing risk to orofacial clefts: today's challenges and tomorrow's opportunities," F1000Research, vol. 5, p. 2800, 2016.

[18] C. P. Savastano, L. A. Brito, A. C. Faria et al., "Impact of rare variants inARHGAP29to the etiology of oral clefts: role of loss-of-functionvsmissense variants," Clinical Genetics, vol. 91, no. 5, pp. 683-689, 2017.

[19] D. Chandrasekharan and A. Ramanathan, "Identification of a novel heterozygous truncation mutation in exon 1 of ARHGAP29 in an Indian subject with nonsyndromic cleft lip with cleft palate," European journal of dentistry, vol. 8, no. 4, pp. 528-532, 2019.

[20] E. J. Leslie, M. A. Taub, H. Liu et al., "Identification of functional variants for cleft lip with or without cleft palate in or near PAX7, FGFR2, and NOG by targeted sequencing of GWAS loci," American Journal of Human Genetics, vol. 96, no. 3, pp. 397-411, 2015.

[21] A. Butali, P. Mossey, W. Adeyemo et al., "Rare functional variants in genome-wide association identified candidate genes for nonsyndromic clefts in the African population," American Journal of Medical Genetics. Part A, vol. 164, no. 10, pp. 25672571, 2014. 